\title{
Teil V: Forschungseinblicke in studentische Arbeiten
}

\author{
Katrin Bente Karl und Studierende
}

Dieser Teil des Bandes liefert Einblicke in Forschungsarbeiten von studentischen Teilnehmerinnen, die im Zusammenhang mit dem Projekt UnVergessen entstanden sind. Dabei handelt es sich sowohl um Forschungsberichte, die Teil der Projektteilnahme sind als auch um vertiefende Abhandlungen, die aus entsprechend thematisch ausgerichteten Qualifizierungsarbeiten hervorgegangen sind.

\section{Einleitung}

Katrin Bente Karl

Durch die Verankerung an der Universität und die begleitende Rahmung durch universitäre Seminare ist neben den sozialen Zielen eine parallele wissenschaftliche Betrachtung des Themenkomplexes rund um Mehrsprachigkeit im Pflegeheim fester Bestandteil von UnVergessen. Wie bereits im dritten Teil des Bandes ausgeführt, durchläuft auch die wissenschaftliche Fokussierung eine Entwicklung. Wo diese zu Beginn des Projektes erst in Ansätzen verfolgt wurde, nahm sie mit der Förderung durch inSTUDIES ${ }^{\text {PLUS }}$ einen Schwerpunkt im Projekt ein, der bis heute beibehalten und sogar noch vertieft wurde. Die mit der Förderung verbundene und bis heute gebliebene stärkere Öffnung des Projektes für studentische Teilnehmer/innen unterschiedlicher Studienphasen und -gänge führte

K. B. Karl und Studierende ( $\square)$

Seminar für Slavistik/Lotman-Institut, Ruhr-Universität Bochum, Bochum, Deutschland E-Mail: katrin.karl@rub.de 
zu einer größeren Vielfalt an Interessen, Vorkenntnissen und auch mitgebrachten methodischen Kompetenzen, was wiederum zu einer verstärkten Möglichkeit führte, wissenschaftlichen Fragestellungen nachzugehen. Dabei entstehen zweierlei Forschungsarbeiten im Umfeld des Projektes: Zum einen ist die Ausarbeitung einer wissenschaftlichen Fragestellung und deren bestmögliche, meist empirische Umsetzung, Auswertung und Interpretation Teil der zweiten Projekthälfte. Zum Abschluss der Teilnahme soll darauf basierend ein Forschungsbericht eingereicht werden, der diese Arbeit dokumentiert. Zum anderen sind aus der Teilnahme am Projekt heraus weiterführende Arbeiten entstanden: Ehemalige Teilnehmer/innen entwickelten durch die Erfahrungen im Projekt ein tieferes Interesse an der Thematik und widmeten dieser ihre Abschlussarbeiten. So sind bislang drei Bachelor-Arbeiten und zwei Masterarbeiten aus UnVergessen hervorgegangen, eine Dissertation zu diesem Themenbereich ist im Entstehen. Welche wissenschaftlichen Potenziale in der Projektkonzeption liegen, wurde bereits weiter oben dargestellt (vgl. Karl in diesem Band „Das wissenschaftliche Potenzial des Projektes"). An dieser Stelle sollen nun vier studentische Fragestellungen als konkrete Beispiele für die Entwicklung des jeweiligen Forschungsinteresses, Wahl der Methode und Skizze der entsprechenden Auswertungsmöglichkeiten angeführt werden. Alle vier Arbeiten konzentrieren sich auf unterschiedliche Facetten der Testung oder auch Beschreibung der Interaktion mit Menschen mit Demenz und decken dabei die russische und polnische Sprache ab. Ich möchte im Vorfeld darauf hinweisen, dass diese Ausführungen nicht als umfassende wissenschaftliche Ausarbeitung des jeweiligen Aspektes zu betrachten sind, sondern dazu konzipiert sind, Einblicke in den laufenden wissenschaftlichen Prozess zu liefern. $\mathrm{Zu}$ diesem Zweck werden unterschiedliche Stationen der wissenschaftlichen Vorgehensweise thematisiert und dabei ein besonderes Augenmerk auf die Herausforderungen gelegt, die mit der speziellen Thematik bzw. gewählten Vorgehensweise einhergehen. Es liegt uns besonders am Herzen, dass nicht auf nüchterne Weise wissenschaftliche Erkenntnisse vermittelt werden, sondern jeder Ausführung eine bestimmte Botschaft innewohnt, die nach außen als Zeichen dessen gesendet werden soll, dass es zahlreiche Ansätze gibt, mit Menschen mit Demenz in eine positive Beziehung zu treten. Auch hier zieht sich also ein konstruktiver und gerade nicht defizitorientierter Ansatz durch, der als Anregung für den Umgang mit Menschen mit Demenz angesehen werden soll. Dieser Ansatz knüpft an den letzten Erfahrungsbericht im vorherigen Kapitel an. Passenderweise bildet dieselbe Autorin den Auftakt: Aldona Rzitki beschreibt die besonderen Herausforderungen, die sich bei der Testung von Menschen mit Demenz ergeben und lässt uns an ihren Überlegungen und der Entwicklung einer Pilotstudie teilhaben. Die anschließenden Ausführungen von Yvonne Behrens fokussieren ebenfalls die 
Problematik der Testung und zeigen, wie mithilfe einer spielerischen Herangehensweise erfolgreich Daten gesammelt werden und zugleich die Testpersonen eine entspannte, abwechslungsreiche und angenehme Gesprächssituation genießen können.

Im dritten Beitrag untersucht Natalja Friesen die besondere Kommunikationssituation eines russischsprachigen, an Demenz erkrankten Mannes, der in einem deutschsprachigen Pflegeheim lebt. Sie fokussiert sich dabei vor allem auf die Frage, wie die Interaktion zwischen ihm und seinen deutschsprachigen Pflegekräften zu beschreiben ist und schlägt damit einen Bogen zu den Ausführungen im zweiten Teil dieses Buches (vgl. Karl in diesem Band ,Was bedeutet Pflegebedürftigkeit für Mehrsprachige?"). Im letzten Artikel von Erika Erhardt tritt eine bereits bekannte Person wieder auf. Wir treffen Frau Schneider wieder: eine russischsprachige demenziell veränderte Person, die seit mehreren Jahren von UnVergessen begleitet wird. Hier zeigt Erika Erhardt, wie es ihr gelungen ist, eine scheinbar verstummende Frau mithilfe einer Nacherzählungsaufgabe zum Sprechen zu bringen.

Diese vier Ausführungen bieten Einblicke in die Möglichkeit, die Erfahrungen, die aus der Projektteilnahme entstehen, wissenschaftlich aufzuarbeiten und in entsprechende Fragestellungen, Methoden und Erkenntnisse zu überführen. Einige der eingesetzten Methoden überschneiden sich dabei und konnten so bereits in unterschiedlichen Konstellationen - hier jedoch immer mit demenziell veränderten Menschen - eingesetzt und erprobt werden. So wächst durch die einzelnen studentischen Arbeiten das Methodenrepertoire und die Erfahrung, welche der Methoden in dieser besonderen Testsituation von Menschen mit Demenz sich bewähren und welche evtl. auch nicht. Als besonders vielversprechend zeigt sich dabei der Einsatz der Nacherzählung einer Bildergeschichte. Diese Methode ist bereits in linguistischen Arbeiten vielfach eingesetzt worden und hat auf unterschiedliche Probandengruppen Anwendung gefunden, eine Übertragung auf Demenzerkrankte ist daher naheliegend (und auch in einigen Studien bereits durchgeführt worden, vgl. hierfür z. B. Ash et al. (2013) und die frühen Studien von Hier et al. (1985) oder Nicholas et al. (1985)). Dabei zeigte sich jedoch im Laufe einiger studentischer Arbeiten, dass sich nicht jede Bildgeschichte als Grundlage gleichermaßen gut eignet. Sie sollte weder zu kurz (wir versuchten es mit lediglich zwei Bildern) noch zu undeutlich gezeichnet sein (wir wählten in einem Projektdurchlauf die Geschichte „Der Sturz ins Tulpenbeet“, die jedoch von vielen der Proband/innen nicht gut genug visuell verarbeitet werden konnte). Letztlich wählten wir die MAIN-Bildergeschichten, die von Gagarina und Kolleg/innen $(2012,2019)$ für den Einsatz der Testung von bilingualen Kindern entwickelt wurden und pilotierten diese für die Testung von Menschen mit 
Demenz. Einblicke in die Ergebnisse dazu finden sich in den Ausführungen von Rzitki und Erhardt, eine kürzlich entstandene Masterarbeit (Friesen, 2020) widmete sich zudem der genaueren Darstellung dieser Methode. Hier scheint ein fruchtbarer methodischer Ansatz gefunden zu sein, dem es nun in weiteren Arbeiten nachzugehen gilt. Und genau in diesem Gedanken liegt der Sinn der folgenden Darstellungen: Sie sollen Anreize schaffen, sich mit der Thematik wissenschaftlich auseinanderzusetzen und dabei nicht die Herausforderungen und das besondere Feingefühl bei der Testung von Menschen mit Demenz aus dem Blick zu verlieren. Damit möchten wir skizzieren, wie eine Forschung aussehen kann, die soziale Aspekte verinnerlicht hat.

\section{Über das Erheben empirischer Sprachdaten bei älteren Menschen - Einblicke in Problematiken und deren Lösungsansätze}

\section{Aldona Rzitki}

Liest man von wissenschaftlichen Methoden und Auswertungen, stellt man sich schnell Personen vor, die mit weißen Kitteln und Schutzbrillen durch sterile Labore laufen. Dies ist jedoch nur ein Teil der Wissenschaft. In der Realität ist sie vielseitiger, denn die Wissenschaft kann auch fernab von Laboren betrieben werden und dabei ohne Reagenzgläser und Chemikalien auskommen. Sie kann sogar auf etwas sehr Elementarem basieren, das in der Regel ohne größere Hilfsmittel durchführbar ist: auf Gesprächen und Erzählungen.

In diesem Beitrag wird es darum gehen, vorzustellen, wie ich empirische Daten für eine wissenschaftliche Pilotstudie im Bereich der Linguistik eingeholt habe. Die für die Pilotierung rekrutierten Probandinnen sind älter als 65 Jahre alt, monolingual und weiblich. Inwiefern dies mein empirisches Vorgehen prägt und im Vergleich zu Testungen anderer Probandengruppen andersartig macht, möchte ich mit diesem Beitrag darstellen. Der Fokus liegt dabei nicht auf der Auswertung der Daten oder den Ergebnissen, sondern vielmehr in der Planung und den Details der Erhebung. Diese möchte ich Schritt für Schritt darstellen und damit Einblicke geben in die wissenschaftliche Arbeit und die zugrunde liegenden Überlegungen von Linguist/innen. 


\section{Thema}

$\mathrm{Zu}$ Beginn jeder Studie steht das Forschungsinteresse. Wenn man erst ein grobes Thema hat, für das man sich interessiert, ergeben sich viele Folgeüberlegungen, die es zu berücksichtigen gilt. Mein Forschungsinteresse gilt der Sprache älterer Personen mit Demenz. Mit einer Bildnacherzählungsaufgabe möchte ich untersuchen, welche Unterschiede sich in der mündlichen Sprachproduktion auf semantischer Ebene zwischen Demenzerkrankten und altersgleichen, kognitiv gesunden Personen ergeben.

Nachdem ich dieses Forschungsdesign im Groben erstellt und mich in die entsprechende Grundlagen- und methodische Literatur eingelesen hatte, musste ich aber feststellen, dass ich mir gar nicht sicher sein kann, ob die Studie, so wie ich sie mir vorgestellt habe, funktioniert. Das ist nichts Ungewöhnliches, da man bei seiner ersten Planung nicht an jedes Detail denken kann. Deshalb werden sogenannte Pilotstudien durchgeführt, bei denen man sein Studiendesign mit einer kleineren Proband/innen-Anzahl auf die Probe stellen kann. Und so galt auch für meine Arbeit zunächst die Aufgabe, einen Testdurchlauf der Methode umzusetzen. Der Fokus lag dabei darauf zu schauen, ob die Methode dafür geeignet ist, Sprachdaten älterer Menschen zu erheben, die sich auf den linguistischen Bereich der Semantik beziehen. Für diesen Zweck wurden kognitiv gesunde Personen ausgewählt. Dies zum einen aus dem Grund, da bei der Hauptuntersuchung auch dieser Personenkreis betrachtet werden soll und zum anderen aus der pragmatischen Überlegung, dass, wenn die Methode bei kognitiv gesunden Menschen nicht funktioniert, sie aller Voraussicht nach auch nicht bei demenziell Erkrankten umzusetzen ist. Andersherum könnte man diese Prognose nicht mit gleicher Sicherheit formulieren. Das Ziel des Testdurchlaufes liegt darin zu schauen, ob altersgleiche monolinguale und kognitiv gesunde Proband/innen die gestellten Aufgaben grundsätzlich lösen können.

\section{Kognitive Fähigkeiten und kritische Reflektion des des Mini-Mental-Status-Test (MMST)}

Da ich mich somit in meinem Testdurchlauf mit kognitiv nicht eingeschränkten Personen beschäftigte, bedarf es zunächst einer Einschätzung des kognitiven Zustandes der Proband/innen. Viele Demenzdiagnosen, die von Hausärzt/innen getroffen werden, beruhen auf dem MMST, der 1975 von Folstein, Folstein und McHugh entwickelt und 1990 von Kessler, Denzler und Markowitsch ins Deutsche übertragen wurde (Meitner, 2019). Der MMST gilt als der in Deutschland am 
häufigsten angewandte psychometrische Kurztest und soll eine Demenz diagnostizieren. Aus wissenschaftlicher Perspektive sehe ich die Diagnostik einer Demenz durch so einen kurzen Test sehr kritisch, da das fehlerhafte Beantworten der Fragen oder Lösen der Aufgaben auch auf andere Einschränkungen hinweisen kann. Es kann zum Beispiel sein, dass eine fehlende Alphabetisierung durch nicht vorhandenen Unterricht in Kriegszeiten zu einem unbefriedigenden Ergebnis in dem Test führt.

Ich habe mich trotzdem dazu entschieden, den MMST vor der eigentlichen Testung mit meinen Probandinnen durchzuführen, um eine grobe Einschätzung ihrer kognitiven Fähigkeiten zu bekommen. Dazu habe ich aber das eigentliche Bewertungsraster geändert und unterscheide nur zwischen „Konnte die Aufgaben gut bewältigen“ und „Konnte die Mehrheit der Fragen nicht beantworten“. Hat jemand in der Testung nur wenige Fehler gemacht, so kann nicht von einer ausgeprägten kognitiven Einschränkung gesprochen werden und die Person wurde als Probandin aufgenommen.

\section{Die Rekrutierung der Proband/innen}

Bei der Rekrutierung von Proband/innen wird es zum ersten Mal praktisch. Man muss mit Menschen in Kontakt treten. Es ist schwierig Personen zu finden, die bei der Studie mitmachen wollen. Vor allem kann es bei strengen Kriterien, die die Proband/innen erfüllen müssen, sein, dass sich zunächst keine passenden Personen finden lassen. Denn je mehr Charakteristika bzw. Variablen erfüllt werden müssen, desto schwieriger gestaltet sich die Rekrutierung. Das kann schnell frustrierend werden. Man sollte für diesen Teil also durchaus etwas Zeit einplanen.

Für meine Erhebungen fragte ich in einer Einrichtung des betreuten Wohnens nach, ob ich bei einem Treffen der Mieter/innen von meiner Studie erzählen kann. Leider ist es häufig so, dass das Personal Erhebungen gegenüber kritisch eingestellt ist. Das ist zwar durchaus schade, aber gut nachvollziehbar. An vielen Stellen gibt es Personalmangel und eine solche Erhebung bedeutet immer einen gewissen Zeitaufwand. Außerdem haben die Institutionen den Menschen gegenüber auch eine gewisse Verantwortung. Wichtig ist es also, eine klare Forschungsethik und Offenheit an den Tag zu legen und diese auch zu kommunizieren, um kein Misstrauen aufkommen zu lassen.

Nach einem Gespräch mit der Leitung der Einrichtung durfte ich mein Anliegen beim wöchentlichen Kaffeetrinken vortragen. Daraufhin meldeten sich einige Probandinnen bei mir, die mitmachen wollten. 


\section{Das Treffen mit den Probandinnen}

Nachdem ich meine Probandinnen rekrutiert hatte, vereinbarte ich mit jeder einen individuellen Termin. Für die Validität und Reliabilität der Ergebnisse ist es wichtig, für ein ruhiges und geeignetes Umfeld zu sorgen. In meinem Fall waren die Testungen bei den Probandinnen in ihrem gewohnten Umfeld bei sich zu Hause. Meistens saßen wir am Esstisch oder sogar auf dem Sofa. Das hatte den großen Vorteil, dass die Personen sich wohl fühlten und zu der ungewohnten Situation nicht auch noch ein ungewohntes Umfeld hinzukam. Das nahm merklich die Nervosität der Teilnehmerinnen, was wiederum den Erhebungen zugute kam. Lediglich das Konzentrationsvermögen durch Ablenkungen ist als Problem aufgefallen. Die Teilnehmerinnen erzählten mir zwischendurch viel zu ihrer Wohnungseinrichtung und Bildern oder Ähnlichem. Ich handhabte es so, dass ich die Personen ausschweifen ließ und anschließend wieder versuchte, auf die Testung zurück zu kommen.

Man sollte sich in solchen Situationen immer wieder bewusst machen, dass man mit Menschen arbeitet. Demzufolge sollte man sich stets fragen, wie man selbst die Befragung erleben wollen würde.

\section{Einverständniserklärung und Aufnahmestart}

Vor Aufnahmestart bekamen die Probandinnen eine Einverständniserklärung, die sie noch einmal genauer über die Aufnahmesituation sowie den Verwendungszweck der Aufnahmen aufklärte. Auch wurden sie über die Anonymisierung der Aufnahmen aufgeklärt, bei der keine Rückschlüsse auf einzelne Personen oder Institutionen gemacht werden können. Natürlich wurden die Probandinnen auch darüber unterrichtet, dass es zu einer Transkription des Datenmaterials kommen kann, die anonymisiert auch in Publikationen verwendet werden könnte.

Nachdem die Teilnehmerinnen einverstanden und bereit waren, nahm ich das Gespräch mit einem Diktiergerät auf. Ich startete die Aufnahme bewusst schon vor den Testungen, um so eine Eingewöhnungsphase zu haben, da die Testung für viele Personen eine Stresssituation darstellt - nicht zuletzt auch für mich selbst. Meiner Erfahrung nach hat man sich aber nach einer kurzen Zeit daran gewöhnt und fast schon vergessen, dass man aufgenommen wird. So kann das Beobachterparadoxon etwas abgeschwächt werden (Albert \& Marx, 2016).

Die Studie an sich sollte immer so gestaltet sein, dass nur das Nötigste abgefragt wird und die Testungen auf die wichtigsten Dinge reduziert werden, da 
sich die Proband/innen sonst schnell wie in einem Verhör fühlen könnten oder schlichtweg durch zu viele Aufgaben demotiviert werden.

In der Regel wird zusätzlich noch ein Fragebogen ausgehändigt, der die biografischen Daten der Teilnehmenden einholt. In meinem Fall teilte ich diesen nach der eigentlichen Testung aus. Wichtig ist auch hier, dass alle für die Erhebung relevanten Faktoren abgefragt werden. Man sollte sich aber unbedingt darüber Gedanken machen, welche Variablen wirklich benötigt werden, da der Fragebogen möglichst kurz sein sollte.

\section{Verantwortung und Dankbarkeit}

Da der Gesprächsbedarf der Personen sehr hoch war, gab es vor den Testungen meist ein ausgiebiges Gespräch über alltägliche Themen. Im Durchschnitt betrug die Dauer der Treffen zwei Stunden. Bei einer Probandin verbrachte ich sogar $4 \mathrm{~h}$. Dies lag aber eher am Redebedarf der Personen und nicht an der Testung. Diese dauerte mit Abzug der individuellen Abschweifungen der Teilnehmerinnen im Schnitt 20 min.

Für mich persönlich ist das Eingehen auf die Person ein sehr wichtiger Punkt, denn von der Bereitschaft der Proband/innen hängt meine Studie ab. Mit den Gesprächen und meinem Interesse an ihrer Person konnte ich ein Stück weit meine Dankbarkeit ausdrücken. Alle Probandinnen freuten sich merklich über meinen Besuch und erzählten mir sehr viel aus ihrem Leben. Am Ende sagten mir alle Teilnehmerinnen, dass sie Spaß hatten und jeder Zeit wieder mitmachen wollen würden.

\section{Bildnacherzählungsaufgabe und abschließende Fragen zur Geschichte}

Aber jetzt zur eigentlichen Testung: Ich ließ die Probandinnen eine von Gagarina und Kolleg/innen $(2012$, 2019) konzipierte Bildergeschichte nacherzählen, die unter dem folgenden Link einsehbar ist: https://www.leibniz-zas.de/de/public ations/schriftenreihe/zaspil/zaspil-56/main-start/

Ursprünglich sind die Bilder für Studien mit Kindern konzipiert worden. Auch für Erwachsene ist - ohne jegliche Wertung - diese Bildergeschichte angemessen, da man davon ausgehen kann, dass alle Beteiligten die abgebildeten Gegenstände und Tiere kennen. So ist der Vergleich unabhängig von dem Bildungsniveau der Teilnehmerinnen gegeben. Gleichzeitig sorgen die ansprechende Gestaltung und 
kurze Dauer der Geschichte dafür, dass die Konzentration bei der Durchführung konstant bleibt. Gerade wenn man an spätere Testungen mit Demenzerkrankten denkt, wird dieser Faktor noch wichtiger.

Die Probandinnen bekamen eine ausgedruckte Variante der Bildergeschichte, bei der die Abbildungen alle nebeneinander angeordnet sind. Zunächst durften die Teilnehmerinnen die Bildergeschichten, so lange wie sie wollten, anschauen, um zu sehen, ob der Zusammenhang verstanden wird und ob alle Elemente der Bildergeschichte gut zu sehen sind. Dies ist besonders wichtig, da das Sehvermögen einiger älterer Personen eingeschränkt sein könnte. Hätten die Teilnehmerinnen nämlich einzelne Elemente der Geschichte nicht erkennen können, so hätte es die Ergebnisse der Studie verfälschen können.

Nachdem die Probandinnen sich mit der Geschichte vertraut gemacht haben, wurde die Bildergeschichte zusammengefaltet, sodass nur die ersten beiden Bilder zu sehen waren. Sie bekamen die Anweisung zu erzählen, was sie auf den Bildern sehen, und daraus eine Geschichte zu formulieren. Nachdem der Arbeitsauftrag für die ersten beiden Bilder fertig gestellt war, wurden die nächsten zwei aufgeklappt und anschließend die nächsten beiden, bis die Bilderabfolge zu Ende beschrieben wurde und die Geschichte wieder vollends vor den Probandinnen lag. Die Geschichte lag dabei so vor ihnen, dass ich selbst die Geschichte nicht sehen konnte, sodass der Eindruck entstehen sollte, dass sie mir eine neue Geschichte erzählen und ich diese nicht kenne.

Im Anschluss daran lobte ich als Versuchsleiterin die Leistung und das Engagement der Probandinnen. Unabhängig davon, ob das Erzählen der Geschichte, gemessen an den Standards in Gagarinas Standards et al. (2012, 2019) erfolgreich war, oder Lücken aufwies. Wichtig ist, dass die Probandinnen sich keinesfalls bloßgestellt fühlen sollen, wenn mal etwas nicht gut geklappt hat. Die Personen sollen sich nämlich zu jedem Zeitpunkt wohl fühlen und Spaß bei den Erhebungen haben.

Wichtig sind dabei an dieser Stelle zwei Dinge. Wenn man zu viel lobt und bestärkt, kann es sein, dass die Proband/innen es nicht ernst nehmen. Man muss also ein Maß finden, um die Bestärkung natürlich klingen zu lassen. Das ist eine Sache, die man während der Erhebung im Hinterkopf behalten sollte. Des Weiteren ist es wichtig, dass man bei den Kommentaren und Bestärkungen immer darauf achten sollte, wie viel und was genau man selbst äußert. Es kann nämlich schnell passieren, dass man mit seinen Aussagen die Antworten der Proband/innen vorstrukturiert bzw. beeinflusst. Fragt man zum Beispiel: „Was sehen Sie auf dem Bild?" ist es wahrscheinlich, dass das Gegenüber seine Aussage beginnt mit: „Ich sehe ...“. Möchte man aber (wie in meinem Fall), dass die Probandinnen aus den Bildern eine Geschichte erzählen, wäre das problematisch. 
Es ist daher ratsam, ein Skript zu erstellen und die genauen Formulierungen für die Fragen festzulegen. Auch ein festes Skript zum Ermutigen der Proband/innen kann hilfreich sein. Vor allem sind feste Vorgaben auch hilfreich, damit alle Proband/innen gleiche Testbedingungen haben und man die Ergebnisse so nicht versehentlich beeinflusst. Es muss aber auch immer klar sein, dass man sich nicht auf jede Eventualität vorbereiten kann. Gerade wenn es um so eine offene Methode geht wie in meiner Pilotierung, kann man zwischendurch von einer Rückfrage oder Abweichungen vom eigentlichen Thema überrascht werden.

\section{Fazit}

Mein empirisches Vorgehen ist geprägt von den rekrutierten Probandinnen. Das Material ist so ausgewählt, dass es auch im hohen Alter noch rezipiert werden kann. Die Testungen werden kurzgehalten, und auch die Ausgangssituation während der Erhebung habe ich als eine ganz besondere wahrgenommen.

Man sitzt als Forscherin vor einer älteren unbekannten Person in einer fremden Wohnung. Trotzdem fühlte ich mich jedes Mal sehr willkommen. Die Erhebungen kamen mir nicht wie ,linguistische Tests' vor. Vielmehr war es nach den ersten 10 min so, als würde man sich mit einer guten Bekannten unterhalten. Dabei wurde mir vor, nach und sogar während der Testungen viel über das Leben der Probandinnen erzählt. Man merkte sehr deutlich, dass man sich sehr darüber freute, einer neuen Person von seinem Leben erzählen zu können. Das war eine sehr schöne Erfahrung, für die ich sehr dankbar bin. Es führte mir noch einmal vor Augen, dass die Arbeit von Linguist/innen nicht nur das Erheben von Daten beinhaltet, sondern dass man durch jede Begegnung mit den Menschen auch einen persönlichen Mehrwert generieren kann. Aus dieser Perspektive ist die Pilotierung also sehr erfolgreich gewesen.

Auch für mein Forschungsvorhaben erwies sich meine Methodik als zielführend. Durch die Auswertung der Aufnahmen habe ich nun eine Basis, auf der ich in kommenden Erhebungen aufbauen kann. Auch das Material erwies sich als gelungen, sodass es nun auch für kognitiv eingeschränkte Personen eingesetzt werden kann.

Lediglich bei der Proband/innensuche würde ich die Altersspanne stärker eingrenzen, da mir bei einigen Personen aufgefallen ist, dass sie altersbedingt kognitive Einschränkungen hatten, die nicht durch den MMST erhoben wurden. 


\section{Ausblick der Ergebnisse}

Elizitationsaufgaben (Bildnacherzählungen) können Auskunft über verschiedene linguistische Bereiche geben, während zum Beispiel Bildbenennungstests sich eher auf lexikalische Kompetenzen fokussieren. Die erhobenen Daten der Elizitationsaufgabe können nun in Hinblick auf bestimmte sprachliche Bestandteile untersucht werden. In meinem Fall segmentiere ich die Äußerungen in Aufzählungen, Beschreibungen, (Handlungs-) Darstellungen und Kommentare und unterteile anschließend in stimulusnahe und stimulusferne Informationen. Auf diesen Erkenntnissen aufbauend kann meine geplante Studie mit kognitiv eingeschränkten Demenzerkrankten durchgeführt und mit der Kontrollgruppe kognitiv gesunder Personen abgeglichen werden. Eine mögliche Erkenntnis, die aus einer solchen Studie gewonnen werden könnte, wäre, dass die Gruppe der von Demenz betroffenen Personen mehr Gesamtäußerungen tätigen wird, diese aber einen geringeren Informationsgehalt haben wird. Also eher aus Beschreibung, Kommentar und Rückfragen bestehen wird. In vergleichbaren Studien (Meitner, 2019) wird der Unterschied zwischen den Gruppen in der Handlungsdarstellung am deutlichsten. Diese stellt eine Transferleistung dar, da man das Gesehene in eine Geschichte umwandeln muss. Die Gruppe Demenzerkrankter produziert in vergleichbaren Studien nur halb so viele Handlungsdarstellungen wie die kognitiv gesunde Kontrollgruppe. Außerdem weicht sie häufiger vom Thema ab und produziert mehr für die Bildergeschichte irrelevante Informationen. Die für die Proband/innen neue Geschichte fordert dazu auf, spontane mündliche Äußerungen zu produzieren. Meitner nimmt aufgrund ihrer Ergebnisse an, dass der Gruppe Demenzerkrankter die Verknüpfung der Informationen und damit einhergehend die Interpretation des Materials schwerfällt und die Proband/innen ihre fehlenden Kompetenzen kompensieren, indem sie eher auf Leistungen zurückgreifen, die keinen Transfer der Informationen erfordern oder sogar thematisch abweichen. Ob sich dies bestätigt, wird sich in Folgestudien, die auf dieser hier beschriebenen Pilotierung aufbauen können, zeigen.

\section{Lassen Sie uns ein Spiel spielen!}

\section{Yvonne Behrens}

Wie kann man den Wortschatz von Menschen mit Demenz untersuchen, ohne dass die Proband/innen Stress oder Druck verspüren? In meinem Promotionsprojekt zur Thematik Demenz und Sprache ist mir die Vermeidung solcher Gefühle 
äußerst wichtig. Es galt also, eine Methode zur Untersuchung des Wortschatzes zu finden, welche weniger als Test, sondern viel mehr als Spiel wahrgenommen wird. Daher entschied ich mich für Bildbenennungs- und Bildauswahltests. Diese eignen sich sehr gut, um die Unterschiede zwischen dem Sprachverständnis und der Sprachproduktion bei mehrsprachigen sowie einsprachigen Menschen mit Demenz zu untersuchen. Doch wie laufen diese Tests ab? Wie beginnen die Treffen und wie enden sie? Werfen wir einen Blick darauf, wie meine Proband/innen und ich ein kleines Bilderspiel spielen.

Für die Erforschung des Wortschatzes von Menschen mit Demenz untersuche ich insgesamt drei Gruppen. Die Hauptgruppe sind polnisch-deutsche Mehrsprachige. Die Vergleichsgruppen sind deutsche und polnische Einsprachige. Alle Personen sind an einer Alzheimer-Demenz erkrankt. Die Proband/innen treffe ich in ihrer gewohnten Umgebung: stationäre und teilstationäre Pflegeeinrichtungen. Für die Testung ist es wichtig, dass die Proband/innen kommunikativ sind und gut erhaltene Sehfähigkeiten haben.

Um den Wortschatz der Proband/innen zu untersuchen, habe ich Bildbenennungs- und Bildauswahltests in polnischer und deutscher Sprache erstellt. Sie behandeln Wörter aus dem Allgemeinwortschatz (bspw. Blume) und dem Pflegewortschatz (bspw. Handtuch). Letzterer besteht aus Wörtern, welche während der Morgenpflege von Menschen mit Demenz verwendet werden. Insgesamt verwende ich Begriffe aus den Kategorien Möbel, Körperteile, Tiere, Ernährung und Pflegeutensilien. Die Tests bestehen jeweils aus 15 Bildern plus einem Übungsbild zum Einstieg. Die ausgewählten Wortschätze überschneiden sich lediglich in wenigen Objekten, beispielsweise wózek inwalidzki (,Rollstuhl'). Die Anzahl der Testitems ist an den Boston Naming Test von Kaplan, Goodglas und Weintraub (1983) angelehnt - ein bildlicher Aphasietest, welcher ebenfalls mit von Alzheimer betroffenen Personen durchgeführt wird. Die von mir ausgewählten Objekte werden, wie bei Gagarina et al. (2010) darauf getestet, ob sie sowohl gesprochen (= Sprachproduktion), als auch verstanden (= Sprachverständnis) werden können. Für die Testung der Sprachproduktion werden die Fragen „Was ist das?“ bzw. „Co to jest?“ gestellt. Um das Sprachverständnis zu untersuchen, werden die Fragen „Wo ist...?“ bzw. „Gdzie jest...?“ gewählt. Alle Objekte sind lebensnah und möglichst allgemeingültig dargestellt. So ist beispielsweise darauf zu achten, dass die Objekte nur wenig Muster enthalten. Es wurden Fotografien verwendet, da diese von Menschen mit Demenz gegenüber Zeichnungen besser erkannt werden (Luckabauer, 2011). Bei bilingualen Proband/innen werden die Testungen sowohl auf Deutsch als auch auf Polnisch durchgeführt. Hieraus ergeben sich vier Testungstage. Mit monolingualen 
Proband/innen werden entweder die deutschen oder die polnischen Testungen unternommen. Es ergeben sich demnach zwei Testungstage.

Wie läuft die Testung nun ab? Zunächst werde ich von einer den Proband/innen vertrauten Person vorgestellt. Dies sorgt bei den Proband/innen für mehr Offenheit und auch Vertrauen gegenüber der Testsituation. Das Ziel des Vorhabens wird leicht verständlich erläutert. Auf das Wort „Test“ wird gänzlich verzichtet. Stattdessen werden die Proband/innen gefragt, ob sie Lust haben, ein Bilderspiel zu spielen. Denn die Tests haben einen spielerischen Charakter. Zudem wird angenommen, dass der Begriff „Spiel“ positiver konnotiert ist als das Wort „Test“. Dies sorgt dafür, dass für die Proband/innen weniger Stress entsteht und sie die Testung als zusätzliches Angebot wahrnehmen, in dem sie im Mittelpunkt stehen. Unterstützt wird es dadurch, dass die Tests in einer Eins-zu-Eins-Situation durchgeführt werden. Je nach Proband/in dauern die Testungen unterschiedlich lang. So gibt es Testungen, die bereits nach zehn Minuten abgeschlossen sind. Andere Testungen dauern wiederum bis zu 45 min. Meist liegt dies jedoch nicht an den Tests selbst, sondern eher am Gesprächsbedarf der Teilnehmenden. Nach dem Aufbau von Vertrauen wird als erstes die Sprachproduktion mittels Bildbenennungstest untersucht.

Anschließend folgt eine kurze Pause, in der sich die Proband/innen erholen können. Diese Zeit wird meist dazu genutzt, um etwas zu trinken und sich zu unterhalten. Da die Testungen überwiegend in den Zimmern der Proband/innen stattfinden, findet sich stets ein Gesprächsanlass: Hochzeitsfotos, Fotografien der Enkelkinder und Haustiere oder auch mal ein besonders schöner Ausblick. In dieser Zeit geht es darum zu entspannen. Gespräche über Objekte aus dem Leben der Proband/innen eignen sich dafür besonders, da ihnen diese sehr vertraut sind. Jedes Objekt hat seine Geschichte und regt zu Erzählungen an. Diese Momente sind für mich als Testerin besonders wertvoll, da in dieser Zeit so viel Lebenserfahrung mit mir geteilt wird.

Als zweites wird das Sprachverständnis mittels des Bildauswahltests untersucht. Abschließend unterhalten wir uns über die Spiele, das Leben und lassen so das Treffen entspannt ausklingen. Die Proband/innen haben bisher durchweg betont, dass die Treffen ihnen viel Freude bereitet haben und sie sich auf das nächste Wiedersehen freuen. Dies erfolgt bei den Testpersonen in der Tat kurze Zeit darauf, da pro Treffen nur zwei Tests in jeweils einer Sprache gemacht werden. Die Testungen werden auf mehrere Tage verteilt, um einerseits die Proband/innen nicht zu überlasten und bei den bilingualen Testpersonen ebenfalls, um die Wörter aus den Tests nicht zweimal am selben Tag in den verschiedenen Sprachen zu testen. Die Objekte werden nämlich sowohl auf Polnisch als auch auf 
Abb. 1 Einblick in die Testung des

Sprachverständnisses: Wo ist die Erdbeere? (Foto: T. Beckschwarte)

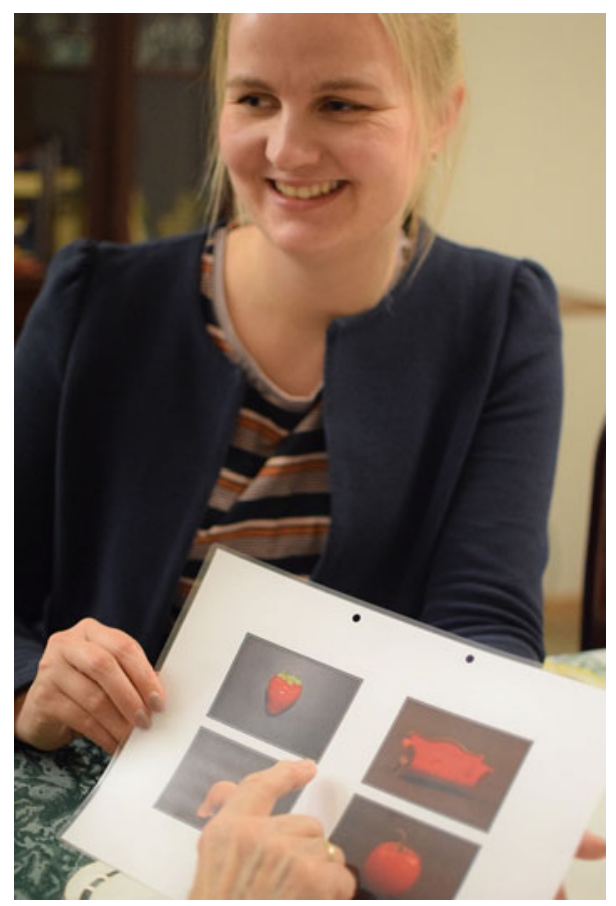

Deutsch getestet. Es handelt sich also um dieselben Objekte. Eine Testung beider Sprachen an einem Tag könnte zu einer Beeinflussung der Ergebnisse führen (Abb. 1).

Die Studien zeigen bisher interessante Ergebnisse. Exemplarisch möchte ich hier auf einige Verhaltensweisen von zwei Proband/innen (VP_2 und VP_22) aus dem Test zum polnischen Allgemeinwortschatz eingehen. Es handelt sich hier um ihre Reaktionen auf winogrona (,Weintraube') und piła (,Säge'). So kann VP_2 das Wort winogrona (,Weintraube') zwar nicht produzieren, erkennt es jedoch als „owoce“ (,Obst'). Diese Unsicherheit in der Benennung wird dadurch überdeckt, dass nicht näher auf das Wort winogrona eingegangen, sondern eher allgemein über die Kategorie Obst gesprochen wird. Mütterlich gibt sie mir den Hinweis „Jedzenie owoców jest ważne!“ (,Obst essen ist wichtig!‘). Die Thematik Obst wird also in den Mittelpunkt gestellt, das Fehlen des Wortes winogrona wird an den Rand gerückt. Eine andere Strategie verwendet VP_22. Diese erkennt das Objekt piła (,Säge') zwar, kann es jedoch nicht benennen. Stattdessen wird das 
Thema des Gebrauchs gewählt. So demonstriert sie den Gebrauch mit der passenden Bewegung und fügt hinzu, dass dieser Gegenstand ,dla mężczyzn“ (,für Männer') ist. Beide Proband/innen erkennen also die Objekte, finden aber nicht das korrekte Wort. Jedoch haben sie Strategien entwickelt, um diesen Umstand zu verdecken. Hierbei handelt es sich um ein typisches Phänomen der ersten Phase von Alzheimer (vgl. Karl in diesem Band „Demenz“). Die Ausführungen der Proband/innen werden wohlwollend bestätigt, damit die Proband/innen ihr Gesicht wahren können und das Vertrauensverhältnis bestehen bleibt.

Die Verwendung des Begriffs „Spiel“ statt „Test“ beweist sich in meinen Testungen als guter Umgang mit meinen Proband/innen. Dies sorgt für eine ruhige, entspannte und auch fröhliche Testsituation, in der viel gesprochen und auch gelacht wird. Ein weiterer Vorteil der lebensnahen Aufmachung der Bildbenennungs- und Bildauswahltests ist, dass die Proband/innen zur Erinnerung an ihre eigene Geschichte angeregt werden. Die bisherigen Ergebnisse sind vielseitig, zeigen aber auch gleiche Tendenzen bei den Teilnehmer/innen. Letzteres zeigt sich besonders in der Verdeckung von Wortlücken. Im Vordergrund meines Promotionsprojekts stehen die wissenschaftlichen Erkenntnisse, jedoch bietet nahezu jedes Treffen auch persönliche Einblicke in eine andere, teils vergangene Welt. Die Kombination dieser beiden Bereiche stellt für mich einen besonders großen Gewinn dar, welchen ich ohne mein Promotionsprojekt wohl kaum bekommen könnte.

\section{Pflegekommunikation in zwei Sprachen: Wie gelingt die Morgenpflege eines demenzerkrankten russischsprachigen Mannes durch deutschsprachige Pflegekräfte?}

Natalja Friesen

\section{Wer ist Konstantin?}

Herr Mironow ${ }^{1}$ lebt in einer Pflegeeinrichtung in Essen. Seit wann er dort lebt, ist allerdings nicht bekannt. Generell weiß man nicht so viel über Konstantin, wie er von allen im Pflegeheim genannt wird. Ursprünglich kommt er aus Kasachstan und kam mit seiner zweiten Frau zusammen nach Deutschland - wann, ist nicht bekannt. Er hat Kinder, die sowohl in Kasachstan als auch in Deutschland leben. Seitdem seine Frau verstorben ist, besucht ihn seine Tochter regelmäßig.

\footnotetext{
${ }^{1}$ Der Name wurde geändert.
} 
All diese Informationen habe ich vom Pflegeheim erfahren, aber nicht von Konstantin selbst, denn er hat Demenz und kann sich nicht mehr daran erinnern. Aufgrund der Erkrankung spricht er kein Deutsch mehr, was ihn sehr belastet, denn eigentlich ist Konstantin ein sehr kommunikativer Mensch und ist gerne in Gesellschaft anderer. An Gesellschaft mangelt es in der Pflegeeinrichtung eigentlich nicht, aber der Verlust der deutschen Sprache stellt eine enorme Barriere dar. Konstantin war oft allein in seinem Zimmer oder befand sich im Speisesaal, wo er aus dem Fenster andere Menschen beobachtete. Sprechen konnte er nur ab und zu mit einer Pflegekraft, die zufällig auch Russisch sprach. Auch andere Pflegekräfte lernten extra für ihn einige russische Ausdrücke, um mit Konstantin eine Beziehung aufbauen und ihn ein wenig aufmuntern zu können, aber eine richtige Unterhaltung konnten diese Gesten leider nicht ersetzen. Außerdem waren die Pflegepersonen stets beschäftigt und konnten sich nicht immer die Zeit für einen Plausch mit Konstantin nehmen. Aus diesem Grund entstand das Projekt UnVergessen für Menschen, die kaum jemanden zu reden hatten. Als Teil des Projektes besuchte ich Konstantin von 2018 bis 2020 regelmäßig. Wir sprachen zusammen, gingen spazieren, aber vor allem spielten wir Karten - das bereitete Konstantin eine besondere Freude. Ich weiß nicht, ob ihn das Kartenspielen an frühere, besondere Momente erinnerte oder einfach an eine Zeit, die er mit seinen Freunden verbracht hatte. Wenn wir spielten, dann lud er andere Pflegeheimbewohner/innen ein teilzunehmen, allerdings auf Russisch - der Sprache, die ihm aufgrund der Demenz noch blieb. Weil die anderen ihn jedoch nicht verstanden, lehnten sie ab. Das war schwer anzusehen, weil es so schien, als ob es Konstantin gar nicht bewusst war, dass er auf einer Sprache spricht, die andere nicht verstehen konnte, und er so die Absagen nicht konkret nachvollziehen konnte. Diese Vorkommnisse riefen in mir die Frage hervor, wie überhaupt die Pflegekräfte ihre Tätigkeiten mit Konstantin durchführen, wenn sie nicht die gleiche Sprache sprechen. Welche sprachlichen Methoden oder Strategien werden angewendet, damit sie ihre Arbeit problemlos vollziehen können? Im ersten Teil dieses vorliegenden Bandes wurden bereits grundlegende Definitionen und Erklärungen geliefert. Auf diese möchte ich hier verweisen und greife einige von ihnen in Kürze noch einmal auf. Ebenso wird im zweiten Teil (vgl. Karl in diesem Band) auf die unterschiedlichen sprachlichen Konstellationen in Pflegeheimen eingegangen. Die hier geschilderte Situation gehört zur dort dargestellten Konstellation 2, da es in dem Pflegeheim von Herrn Mironow neben deutschsprachigen Pflegekräften auch russischsprachige gibt, die mit ihm Russisch sprechen. Dabei konzentriere ich mich im Folgenden auf die Interaktion mit deutschsprachigen Pflegekräften, da es mich wissenschaftlich besonders interessierte, wie in einer solchen erschwerten sprachlichen Situation Pflege und Austausch funktionieren. Dazu bedarf es eines kurzen 
Einblicks in die Arbeit der Pflegekräfte und die kommunikativen Fähigkeiten von Konstantin.

\section{Besonderheiten der Pflegeinteraktion}

Konstantin ist an Demenz erkrankt, eine Krankheit, die zu den gravierendsten Sprachbeeinträchtigungen bei einer Person führen kann (vgl. Hamers \& Blanc, 2000, S. 78, für eine ausführlichere Darstellung der Krankheit und ihren Folgen s. Karl „Demenz“ in diesem Band).

Durch die Demenz fühlen sich Menschen oft verängstigt und unverstanden. Bei Betroffenen mit einem Migrationshintergrund treten diese Empfindungen oftmals in verstärkter Form auf. Durch die Demenzerkrankung fühlen sich die Personen mit Gefühlen wie Ungewissheit und Fremdheit konfrontiert, die sie bereits bei der Ankunft in ein fremdes Land empfunden haben (vgl. Demenz und Migration).

Eine der größten Belastungen stellt für demenzerkrankte Migrant/innen der durch die Demenz verursachte Verlust ihrer erlernten Zweitsprache dar. Das führt zu Kommunikationsproblemen, Missverständnissen und einem eingeschränkten Kontakt zur Außenwelt, was sozialen Rückzug und Isolation zur Folge hat (vgl. hierfür Karl „Demenz“ in diesem Band). Oftmals treten dazu noch psychische Probleme auf, da die Betroffenen verstehen, dass sie ihre Zweitsprache nicht mehr beherrschen (vgl. Demenz und Migration).

Fast alle diese Punkte finden sich bei Konstantin wieder. Wie gehen jetzt deutschsprachige Pflegepersonen mit solch einer schwierigen Situation um? Wie kann den Betroffenen ein Gefühl des Vertrauens vermittelt werden und überhaupt eine zwischenmenschliche Beziehung aufgebaut werden, wenn die Kommunikationsteilnehmer nicht dieselbe Sprache sprechen?

Kommunikation und nichtsprachliche Handlungen im Pflegeheim sind empraktisch, das heißt, dass pflegerische Gespräche meistens handlungsorientiert sind. Kommunikation dient dabei zur Durchführung einer Pflegehandlung. Der Gesprächsverlauf orientiert sich meist an dem geistigen Zustand der gepflegten Person. Um das Gespräch so erfolgreich wie möglich gestalten zu können, verwenden Pflegekräfte unterschiedliche Strategien:

- Handlungsorientierte Strategie

Diese Strategie wird bei Bewohner/innen verwendet, die akustische oder mentale Einschränkungen aufweisen und die Gesprächserwartungen nicht uneingeschränkt erfüllen können. Aus diesem Grund werden wenig medizinische Begriffe verwendet und stattdessen vermehrt alltägliche Worte in das 
Gespräch eingebaut. Prinzipiell gilt: je größer die Pflegebedürftigkeit, desto einfacher die Gestaltung der Sprache (vgl. Sachweh, 2000, S. 114).

- Gesichtsschonende Strategie

Diese Strategie wird vor allem dazu verwendet, um ein Gespräch mit den Betroffenen so höflich wie möglich zu gestalten. Hier wird der Imperativ häufig durch Konjunktiv oder Modalverben ersetzt. Außerdem werden Aufforderungen mit bitte oder als höfliche Frage formuliert. Bestätigungssignale wie $\mathrm{mhm}$ oder ach so werden verwendet, um einen normal gelingenden Kommunikationsverlauf zu simulieren, wenn Betroffene unklare Äußerungen produzieren (vgl. Sachweh, 2000, S. 130 ff).

- Secondary Baby Talk Strategie

Unter dieser Strategie versteht man eine starke Vereinfachung der Kommunikation. Der Wortschatz fasst nicht mehr als 100 Wörter und dazu kommt ein überzogener Intonationsverlauf, eine hohe Tonhöhe, mehr Imperative und viele Wiederholungen, besonders von Namen, damit die Aufmerksamkeit der Betroffenen nicht verloren geht (vgl. Sachweh, 2003, S. 154).

- Gesichtsbedrohende Strategie

Bei dieser Strategie kann nicht von einer Strategie per se gesprochen werden, denn dabei handelt es sich nicht um ein strategisches Handeln. Als gesichtsbedrohend werden Unterbrechungen und bestimmte Formen der Adressierung der Bewohner/innen, das Sprechen über die Bewohner/innen in ihrer Anwesenheit sowie das Äußern von direkter oder indirekter Kritik verstanden. Dazu kommt das Imitieren oder Ignorieren von Bedürfnissen der Betroffenen (vgl. Sachweh, 2000, S. 57).

\section{Entwicklung und Umsetzung der Forschungsfrage}

Die kommunikative Situation zwischen nicht gleichsprachigen Gesprächspartnern in der Pflege interessierte mich bereits 2018. Deshalb machte ich es zum Thema meiner Bachelorarbeit. Dort untersuchte ich, mit welchen kommunikativen Strategien die jeweils deutsch- und russischsprachigen Pflegekräfte während der Morgenpflege die inner- und außersprachlichen Aufgaben in der Interaktion mit dem dementen russischsprachigen Bewohner Konstantin durchführen. 2019 führte ich dann die Pilotstudie nochmals durch, um eine quantitative Ergänzung der ursprünglichen Forschungsfrage zu erzielen. In dieser Untersuchung wurde das Augenmerk besonders auf die deutschsprachigen Pflegekräfte gelegt, da dort die größten sprachlichen Komplikationen auftreten. Kurz gefasst interessiert mich 
der Umgang deutschsprachiger Pflegekräfte mit pflegebedürftigen Menschen, die das Deutsche aufgrund einer Erkrankung nicht mehr beherrschen.

Die Zeit, in der sich die Pflegepersonen am meisten mit den Bewohner/innen unterhalten, ist während der Morgenpflege. In dieser Zeit gilt die Konzentration des Personals ausschließlich der zu pflegenden Person. In solchen Momenten ließ sich die sprachliche Interaktion zwischen Konstantin und den Pflegekräften daher am besten beobachten.

So übergab ich ein Aufnahmegerät an das Pflegepersonal, damit sie die Kommunikation während der Morgenpflege aufzeichnen konnten. Ich selbst habe mich in diesem Schritt zurückgezogen, da meine Anwesenheit erstens bei der Morgenpflege unangebracht wäre, und zweitens hätte es die ,natürliche“ Kommunikation immens beeinflusst. Natürlich ist hier in Anführungszeichen angeführt, da man nicht konkret von einer natürlichen bzw. authentischen Kommunikation von der Seite der Pflegekräfte sprechen kann. Die Tatsache, dass die Pflegepersonen wissen, dass sie in dem Moment aufgenommen werden, beeinflusst ihre Art zu sprechen, ob bewusst oder unbewusst. Nichtsdestotrotz war es in diesem Rahmen die einzige Möglichkeit, um die Pilotstudie umzusetzen. Die Aufnahmedauer sollte sich über einen Zeitraum von zwei Wochen strecken.

\section{Ergebnisse}

Am Ende der zwei Wochen sollten insgesamt 14 Aufnahmen analysiert und gegenüberstehend ausgewertet werden. Leider wurde der Ablauf nicht ganz eingehalten, sodass am Ende insgesamt fünf Aufnahmen von fünf deutschsprachigen Pflegerinnen zur Auswertung verfügbar waren.

Die Auswertung der Aufnahmen zeigte, dass alle oben erwähnten Gesprächsstrategien angewendet wurden. Vor allem die verständnissichernden Strategien wurden von allen Pflegerinnen gebraucht. Drei von fünf verwendeten zusätzlich die gesichtsschonende und Secondary Baby Talk Strategie, während eine einzige Pflegerin die gesichtsbedrohende Strategie bei der Durchführung der Morgenpflege verwendete.

Die Strategie der Verständnissicherung äußerte sich in der häufigen Verwendung von Gliederungssignalen wie ja, gut, schön oder okay. Dabei konnte davon ausgegangen werden, dass Konstantin diese Bestätigungswörter verstehen konnte, da er diese ab und zu selbst gebraucht hat. Des Weiteren erfolgte der Gebrauch von Handlungsanweisungen (aufstehen, hinsetzen, hoch, runter) und sehr einfach gestalteten Äußerungen. 
Drei der Pflegerinnen haben sich zusätzlich bemüht, das Gespräch möglichst höflich zu gestalten und verwendeten deshalb die gesichtsschonende Strategie. Sie verwendeten Hörersignale wie $a h a$ und $m h m$, um das Gespräch so real wie möglich aussehen zu lassen, weil sie die meisten Äußerungen von Konstantin nicht verstehen konnten, da er mit ihnen Russisch sprach. Außerdem wurden Aufforderungen häufig mit bitte formuliert.

Am häufigsten wurde jedoch das Secondary Baby Talk verwendet, da diese Strategie gleichzeitig auch die Verständnissicherung miteinbezieht. Vier der fünf Pflegerinnen verwendeten sehr kurze und einfach gehaltene Sätze mit vielen Wiederholungen und der Betonung der Schlüsselwörter. Dazu kommt eine langsamere und deutlichere Aussprache der Schlüsselwörter sowie eine höhere Tonhöhe am Ende der gesprochenen Sätze. Eine Pflegerin spricht extra mit einer kindlichen Intonation mit der Absicht, Konstantin dazu zu bewegen, Deutsch mit ihr zu sprechen, damit sie ihn verstehen kann. Dies gelingt ihr jedoch nicht. Eine weitere Pflegerin verwendet übermäßig viele Imperative, eine sehr einfachgehaltene Sprechweise, um die Morgenpflege durchführen zu können. Außerdem spricht sie ihn häufig mit dem Namen an und gibt ihm sogar einen Spitznamen, um seine Aufmerksamkeit zu halten.

Eine Pflegerin verwendet auch die gesichtsbedrohende Strategie in der Durchführung der Morgenpflege. Sie ignoriert Konstantin, als er nach Essen fragt oder ihr mitteilt, dass ihm kalt ist. Dies stellt eine schwierige Situation dar, da Konstantin seine Fragen und sein Wohlbefinden ausschließlich auf Russisch äußert und die Pflegerin ihn nicht versteht. Sie kann aber sehr wohl die Frageintonation am Ende seines Satzes nachvollziehen und entscheidet sich dafür, diese komplett zu ignorieren, was eine unangenehme Situation schafft. Zum Teil spricht sie bis zu anderthalb Minuten nicht mit Konstantin oder äußert keinerlei Hörsignale, wenn er spricht.

\section{Ausblick}

Die Ergebnisse meiner Pilotstudie sind selbstverständlich nicht repräsentativ, jedoch sollen sie einen kleinen Einblick in die Kommunikation zwischen deutschsprachigem Pflegepersonal und Menschen mit Demenz, die nicht mehr die deutsche Sprache beherrschen, geben.

Bei der Morgenpflege hat sich gezeigt, dass alle vorgestellten Kommunikationsstrategien gebraucht wurden, einige mehr andere weniger. Alle Pflegerinnen versuchen, Konstantin sprachlich zu erreichen und mit ihm zwischendurch ein 
Gespräch aufzubauen. Sie bemühen sich, indem sie ihn loben oder seine Äußerungen mit Bestätigungssignalen erwidern, obwohl sie ihn nicht verstehen. Dabei bemühen sie sich wirklich, ihn verstehen zu können. Dabei tritt Fantasie und Körpereinsatz in den Vordergrund, um nachvollziehen zu können, was Konstantin ihnen mitteilen möchte. Die meisten Pflegerinnen verwenden das Secondary Baby Talk, allerdings ist das kindliche Sprechen mit einem Erwachsenen nicht immer angebracht, weil Konstantin geistig noch recht klar ist. Er spricht nur kein Deutsch mehr. Nichtsdestotrotz sind die Mühen und der Einsatz der Pflegekräfte, sich mit Konstantin so gut, wie es nur geht unterhalten zu können, lobenswert.

Da es bisher wenig Forschung in Bezug auf Mehrsprachigkeit, Kommunikation und Demenz im deutschsprachigen Raum gibt, soll diese Arbeit einen kleinen Anreiz und Ausgangspunkt für tiefergehende Forschung in diesem Feld bieten.

\section{Wie die Linguistik jemanden zum Reden bringen kann: Unterschiedliche linguistische Testverfahren und ihre sprachlichen Reaktionen bei einer russischsprachigen Pflegebedürftigen}

\section{Erika Erhardt}

\section{Schilderung der Konstellation}

Seit Anfang Februar 2019 habe ich Frau Schneider ${ }^{2}$ in einer Pflegeeinrichtung in Bochum regelmäßig besucht. Die meisten dieser Besuche habe ich dabei mit einem Aufnahmegerät aufgezeichnet. Über Frau Schneider sind nicht viele Details bekannt: Zum Zeitpunkt meiner Besuche war sie 69 Jahre alt und war vermutlich in den 80er Jahren nach Deutschland immigriert. Sie hat eine Tochter und mindestens eine Schwester. Dass so wenige Informationen über Frau Schneider vorliegen, liegt an ihrer Demenzerkrankung. Diese Erkrankung hatte Einfluss auf die Vorbereitung meiner Besuche und der Inhalte, die ich mit Frau Schneider besprochen habe. Durch Herrn Stin (er hatte Frau Schneider in der zweiten Runde des Projekts betreut) hatte ich erfahren, dass sie sich gerne Bildbände ansieht. Besonders ein Band über Moskau hat ihr dabei immer gefallen. Bei meinem ersten Besuch in der Pflegeeinrichtung hatte ich diesen Band dabei und zusätzlich noch einige private Fotos von Moskau. Bei diesem ersten Besuch hat Frau Schneider nicht ein Wort mit mir gewechselt. Stattdessen hat sie nonverbal kommuniziert

\footnotetext{
2 Der Name wurde geändert.
} 
und gelächelt oder genickt, wenn ich ihr Fragen stellte. Die mitgebrachten Fotos hatten sie nur wenig interessiert. Vermutlich lag ihr Desinteresse an der Größe der Fotos. Frau Schneider sieht nicht mehr gut und die Fotos waren möglicherweise zu klein. Aus diesem ersten Treffen konnte ich bereits viel schlussfolgern: Frau Schneider spricht wenig bis gar nicht, sie sieht nicht mehr gut und sie erinnert sich nicht mehr an alle Details ihres Lebens. Bei den weiteren Besuchen stellte ich fest, dass Frau Schneider sich nicht an mich erinnerte. Ich war jede Woche eine neue Person für sie. Somit war es schwierig, eine persönliche Bindung aufzubauen, da eine solche Bindung darauf beruht, dass man seinem Gegenüber vertrauen kann. Dies wiederum setzt voraus, dass man sich an seinen Gesprächspartner erinnern kann. Daher konnte bei meinen Besuchen keine Entwicklung auf dieser Ebene beobachtet werden. Andererseits hatte diese Situation einen Vorteil für mein wissenschaftliches Interesse. Forschungsrelevante Tests konnten in mehreren Durchläufen mit Frau Schneider durchgeführt werden, ohne dass ein für sie relevanter Wiederholungseffekt auftreten konnte. Denn eine Woche später bis zum nächsten Besuch hatte sie bereits vergessen, dass ich bei ihr war und wir Bilder oder Aufgaben schon einmal gemeinsam gelöst hatten. Auch wenn sich auf der Erinnerungsebene keine starke Verbindung aufbauen ließ, lässt sich dennoch eine Entwicklung in den Besuchen erkennen. Zwar konnte sich Frau Schneider nicht an mich erinnern, doch ich erinnere mich an ihre Erzählungen. Daher konnte ich sie von Besuch zu Besuch besser einschätzen und ihre Reaktionen deuten. So kam ich auch auf die Methode der Bildergeschichte. Denn mir wurde im Laufe der Besuche bereits klar, dass Frau Schneider nicht auf das Sprechen verzichtet, weil sie es nicht mehr kann. Sie muss lediglich gefordert werden. Das heißt, wenn ich Aufgaben dabeihatte, wie Bildbenennungen, hat sie sehr gut mitgemacht. Nur aus eigener Initiative wollte Frau Schneider auch weiterhin nicht sprechen. Vor diesem Hintergrund ergab sich die Idee zur Forschungsmethode und den Bildergeschichten von Gagarina und Kolleg/innen (vgl. Gagarina et al. 2012, 2019).

\section{Entwicklung der Forschungsfrage}

Zunächst hatte meine Forschung ein ganz anderes Ziel. Untersucht werden sollte eine grammatische Kategorie - der Verbalaspekt -, die fester Bestandteil des Russischen ist, dem Deutschen aber fehlt (vgl. Clasmeier, 2015, S. 27). Hier sollte untersucht werden, ob Frau Schneider unter der besonderen Konstellation der Demenz und der Migration das Wissen um diese Kategorie abgebaut hat (vgl. 
Neppert, 2010, S. 12). Um dies zu untersuchen, führte ich verschiedene linguistische Tests mit ihr durch. Das Ergebnis dieser Tests war, dass Frau Schneider die grammatische Kategorie des Verbalaspekts problemlos beherrschte. Bei den Untersuchungen fiel jedoch etwas anderes auf: In offenen Gesprächen äußerte sich Frau Schneider kaum, sie antwortete auf Fragen mit 1-2 Wörtern oder gar non-verbal und stellte nie eigene Fragen. Auch sprach sie nie aus eigener Initiative, sondern antwortete lediglich auf Fragen. Besonders auffällig wurden ihre Sprachfähigkeiten bei der Nacherzählung einer Bildergeschichte: Die sonst ,wortkarge" Frau Schneider begann ihre Erzählung mit einer für russische Märchen typischen Redewendung und erzählte die Geschichte in ganzen Sätzen. Dabei kam mir ein anderer Gedanke: Frau Schneider kann durchaus kommunizieren, sie hat die Fähigkeit dazu und die damit verbundenen grammatischen Strukturen nicht abgebaut, sie braucht lediglich einen Anreiz zum Sprechen. Das könnte bedeuten, dass ihre Kommunikationsfähigkeit unter der Demenzerkrankung gelitten hat. In diesem Moment war meine Forschungsfrage geboren: Wie können verschiedene linguistische Tests die Kommunikationsfähigkeit von Menschen mit Demenz an die Oberfläche bringen und im vorliegenden Fall ganz konkret die Kommunikationsfähigkeit der russischsprachigen Frau Schneider zeigen?

Die Tests, die ich zuvor mit Frau Schneider durchgeführt hatte, waren auf verschiedene Bereiche der Sprache abgestimmt. Bei einigen Aufgaben ging es um die Produktion von Sprache, bei anderen um das Verständnis. Die Aufnahmen sollen im Nachhinein dazu dienen, das Gesprächsverhalten von Frau Schneider zu untersuchen und zu vergleichen, wie ihr Redeanteil aussieht bei offenen Gesprächen im Kontrast zu Gesprächen, die Aufgaben beinhalten.

\section{Umsetzung der Forschungsfrage}

Da meine ursprüngliche Forschungsfrage eine andere war, habe ich unterschiedliche linguistische Tests mit Frau Schneider durchgeführt, um generell einen Eindruck zu gewinnen, wie und ob sie überhaupt aktiv teilnimmt. Da sie bei meinem ersten Besuch nicht ein Wort gesagt hatte, wäre es keine Überraschung gewesen, hätte sie auch bei den Tests geschwiegen. Angefangen habe ich mit einem Bildauswahltest, der die Rezeption der Proband/innen untersucht. Bei diesem Test habe ich Frau Schneider ein Blatt mit vier verschiedenen Items gezeigt, wobei sie auf das von mir genannte Wort zeigen sollte. Dies bereitete ihr keinerlei Schwierigkeiten und sie löste die Aufgabe problemlos. Sie ließ sich nicht durch ähnlich aussehende oder ähnliche klingende Gegenstände ablenken. Ebenso reibungslos verliefen die Tests zum Sprachverständnis und zur Bildbenennung. 
Auch hier löste Frau Schneider die Aufgaben ohne Probleme und reagierte sehr gut auf die präsentierten Reize. Diese ersten Ergebnisse ermutigten mich, weitere Versuche mit Frau Schneider durchzuführen. Überrascht hatte sie mich bei einem Assoziationstest, bei dem ich ihr eine Kategorie, z. B. „Farben“ nannte und sie mir daraufhin alle Farben aufzählte, die ihr einfielen, und das nicht nur auf Russisch, sondern auch auf Deutsch! Das zeigte mir, dass Frau Schneider das Wissen der Wörter (in beiden Sprachen!) nicht abgebaut hatte, sondern lediglich einen Anreiz braucht und gefordert werden muss. Dies wurde bei einer weiteren Aufgabe besonders deutlich, die mich, wie bereits oben dargestellt, zum Umdenken meiner Forschungsfrage brachte. Bei der sogenannten „MAIN Geschichte“ von Gagarina und Kolleg/innen (Gagarina et al., 2012, 2019, vgl. hierfür auch den Beitrag von Rzitki in diesem Band) handelt es sich um verschiedene Bildergeschichten, die aus sechs einzelnen Bildern bestehen. Diese sind leicht verständlich und sehr gut für Proband/innen mit Demenz geeignet, da die Aufmerksamkeitsspanne bei diesen Personen eingeschränkt ist und sie bei einer zu langen Bildergeschichte vermutlich den Anfang vergessen würden.

Die einzelnen Bilder der Geschichte habe ich so gefaltet, dass Frau Schneider nur die ersten beiden Bilder sehen und sich besser auf diese konzentrieren konnte. Sobald Frau Schneider sie beschrieben hatte, deckte ich die folgenden beiden Seiten auf, bis sie die gesamte Geschichte sehen konnte. Als Aufgabenstellung erklärte ich Frau Schneider, dass sie die Geschichte so erzählen soll, als würde sie sie einem Kind erzählen. Da die Aufmerksamkeitsspanne von Frau Schneider relativ kurz ist, musste ich zwischendurch Fragen stellen, wenn ich merkte, dass die Gesprächspause zu lang wurde. Es reichte völlig aus zu fragen, was auf dem nächsten Bild geschieht, und Frau Schneider sprach direkt weiter.

\section{Ergebnisse}

Zunächst begann ich meine Analyse in Hinblick auf die erwähnte grammatische Kategorie, die fester Bestandteil des Russischen ist, dem Deutschen aber fehlt. Dabei stellte ich fest, dass Frau Schneider diese Kategorie des Verbalaspekts einwandfrei anwandte. Natürlich kann mit einem einzigen Test kein Urteil über ihre gesamte Sprachkompetenz gefällt werden. Die Ergebnisse deuten jedoch in eine bestimmte Richtung.

Interessanter war jedoch ein anderer Faktor: Frau Schneider hat in den Wochen zuvor bei den Besuchen kaum bis gar nicht gesprochen. Aus eigener Initiative sprach sie gar nicht und antwortete lediglich (sporadisch) auf Fragen. Als sie jedoch darum gebeten wurde, die MAIN Geschichte zu erzählen, sprach sie zum 
ersten Mal im Laufe der Besuche in längeren Sätzen und verwendete Idiome, die typisch für die Erzählung eines russischen Märchens sind. Um diesen deutlichen Unterschied in ihren Aussagen zu veranschaulichen, soll an dieser Stelle ein kurzer Dialog in Form eines Transkripts ${ }^{3}$ gezeigt werden, der bei einem Besuch im April 2019 entstand. Es ist wichtig zu wissen, dass der folgende Dialog vor der MAIN Geschichte aufgenommen wurde, um einen Eindruck davon zu bekommen, warum die Erzählung der Bildergeschichte solch eine Überraschung darstellt.

Transkript 1: Dialog zwischen E. Erhardt und Frau Schneider

Dauer der Audioaufnahme: 1:11 Minuten

Sprecher/innen: $\quad$ Frau Schneider (FS) - russischsprachige demenzerkrankte Bewohnerin eines Pflegeheims.

Erika Erhardt (EE) - studentische Teilnehmerin am Projekt UnVergessen

\section{Transkription}

EE ja mogu pokazat' vam moskvu zimoj ili letom. (Pause) kakie vam lučše?

FS letom.

EE letom, da. (2 Sek. Pause) aaa, vot (gedehnt)(Pause) ja byla dva goda nazad v moskve letom. ja byla tam na stažirovke v bjuro, v ofise (Pause) i (gedehnt) tam ja delala očen' mnogo fotografii. ich ja mogu vam pokazat'. (Pause) esli vam interesno, da?

FS (nickt)

EE vot smotrite (Pause) zdes' na zadnem fone bol'šoj teatr (2 Sek. Pause) vy byli kak-to $\mathrm{v}$ moskve?

FS (nickt)

EE da (Pause) zimoj ili letom?

FS letom

EE letom, da (2 Sek. Pause) i byla chorošaja pogoda?

FS teplo

EE teplo da (3 Sek. Pause) vy byli odni v moskve ili s kem-to?

FS mama

EE s mamoj da (2 Sek. Pause) a vy eščë malen'kie byli?

FS net bol’šaja vzroslaja

\footnotetext{
${ }^{3}$ Die hier gewählte Form der Wiedergabe ist an die Zwecke dieser Ausführungen angepasst und entspricht nicht den gängigen wissenschaftlichen Konventionen. Eine ausführliche Darstellung der gewählten Niederschrift findet sich in Teil II dieses Buches.
} 
EE vzroslaja uže da

\section{Übersetzung}

EE ich kann ihnen moskau im winter oder im sommer zeigen. (Pause) was mögen sie lieber?

FS im sommer.

EE im sommer, ja. (2 Sek. Pause) ah, hier (gedehnt). ich war vor zwei jahren in moskau im sommer, ich habe dort ein praktikum in einem büro gemacht, in einer geschäftsstelle, (Pause) und ich habe dort sehr viele fotos gemacht. ich kann sie Ihnen zeigen, (Pause) wenn sie interessiert sind, ja?

FS (nickt)

EE hier, sehen sie, (Pause) da ist das bolschoi-theater im hintergrund. (2 Sek. Pause) waren Sie schon mal in moskau?

FS (nickt)

EE ja. (Pause) im winter oder im sommer?

FS im sommer.

EE im sommer, ja. (2 Sek. Pause) und war das wetter gut?

FS warm.

EE warm, ja. (3 Sek. Pause) waren sie allein in moskau oder mit jemandem?

FS mama.

EE mit der mutter, ja. (2 Sek. Pause) waren sie noch klein?

FS nein. groß, erwachsen.

EE bereits erwachsen, ja

Dieser Dialog zwischen Frau Schneider und mir ist sehr typisch für die Unterhaltungen, die während der Besuche geführt wurden. Aus eigener Initiative hat sie bei keinem der Besuche gesprochen. Auch auf Fragen hat sie zum Teil nur mit einem Nicken oder Kopfschütteln geantwortet oder gar nicht reagiert. Wenn sie verbal auf eine Frage geantwortet hat, dann geschah dies sehr knapp, nie in längeren Sätzen. Daher ist es so erstaunlich, dass Frau Schneider bei der Erzählung der Bildergeschichte genau dies tat: Sie sprach in längeren Sätzen. Zwar musste sie an einigen Stellen zum Weitererzählen aufgefordert werden, kam dieser Aufforderung jedoch sofort nach und sprach weiter. Besonders auffällig ist der Einstieg in die Geschichte. Frau Schneider hat sofort verstanden, dass die Bildergeschichte wie ein Märchen für Kinder erzählt werden soll und hat die typische Wendung gewählt: 
Transkript 2: Beginn der Nacherzählung einer MAIN-Geschichte von Frau Schneider

Dauer der Audioaufnahme 31 Sekunden

Sprecher/innen

Frau Schneider (FS) - russischsprachige demenzerkrankte Bewohnerin eines Pflegeheims,

Erika Erhardt (EE) - studentische Teilnehmerin am Projekt UnVergessen

\section{Transkription}

FS v nekotorom carstve v nekotorom gosudarstve (Pause) žil-byl odin dubok

EE mhm

FS byl on tichij

EE mhm

FS ptički rešili žit' na nëm

EE mhm

FS tri ptički (2 Sek. Pause) odna iz nich byla letučaja sil'no

EE mhm

FS sil'no letala bystro

\section{Übersetzung}

FS in irgendeinem königreich, in irgendeinem staat (Pause) gab es ${ }^{4}$ eine kleine Eiche.

EE mhm.

FS sie war ruhig.

EE mhm.

FS die vögel beschlossen, sich darauf niederzulassen.

EE mhm.

FS drei Vögel. (2 Sek. Pause) einer von ihnen war ein starker flieger.

EE mhm.

FS stark flog er schnell

Solch ein Beginn ist klassisch für russische Märchen, wenn es um einen Zaren oder ähnliches geht.

\footnotetext{
${ }^{4}$ Diese Wendung entspricht einem typischen Beginn eines russischen Märchens und ist damit vergleichbar mit der deutschen Wendung: Es war einmal...
} 
Der markante Unterschied zwischen den beiden Aufnahmen brachte mich zum Nachdenken. Schließlich zeigte sich in der Nacherzählung sehr klar, dass Frau Schneider ihre Sprachfähigkeiten im Russischen nicht abgebaut hat. Diese scheinen noch gegeben zu sein, sie wurden eventuell in den vorigen Besuchen nur nicht durch ,passende“ Fragen oder Aufgaben zum Vorschein gebracht. Möglich ist, dass Frau Schneider einige soziale Kompetenzen abgebaut hat, die zum Beispiel für die Kommunikation mit ihren Mitmenschen zuständig sind. Das heißt, dass die sprachlichen Strukturen trotz der genannten Einflussfaktoren intakt sind. Lediglich die Kommunikationsfähigkeiten wurden bei ihr abgebaut. Dies wurde vor allem bei einem Austausch mit Herrn Funk deutlich, der Frau Schneider in der ersten Runde des Projekts UnVergessen betreut hatte (vgl. hierfür auch den Poly$\log$ von Golbek in diesem Band). Er berichtete davon, dass Frau Schneider sich aktiv mit ihm unterhielt und gerne Diskussionen über Politik führte. Diese Information lässt vermuten, dass die Demenz bei ihr im Laufe der letzten zwei Jahre vorangeschritten ist und ihre Fähigkeiten zur Kommunikation darunter gelitten haben.

\section{Diskussion und Ausblick}

Das Ergebnis meiner Studie ist nicht repräsentativ, da Aufnahmen und Beobachtungen lediglich einer Person vorliegen. Dies liegt an den aufwendigen Vorarbeiten, bei denen ich zunächst in Erfahrung bringen musste, welche Methode sich am besten für die gegebene Fragestellung und für Frau Schneider eignet. Jedoch bieten diese Aufnahmen einen Einblick in ihre sprachlichen und kommunikativen Fähigkeiten. Sie zeigen, dass die sprachlichen Strukturen, insbesondere die grammatische Kategorie des Verbalaspekts, auch trotz ihrer Erkrankung in ihrem Gehirn verankert sind. Dies zeigten unterschiedliche Tests, die sie problemlos - wenn auch z. T. wortkarg - meisterte. Diese Verankerungen wurden weder durch die Migration von Frau Schneider noch durch ihre fortschreitende Demenzerkrankung beeinträchtigt. Da die grammatischen Kategorien bereits in der frühen Kindheit erworben werden, lässt sich daher vermuten, dass sie aufgrund des frühen Erwerbs nicht (so schnell) abgebaut werden. Im Kontrast dazu fiel die Erzählung der MAIN Geschichte dadurch auf, dass Frau Schneider zum ersten Mal während meiner Besuche in längeren und teils ganzen Sätzen gesprochen hat. Bei den anderen Besuchen hat sie entweder gar nicht gesprochen, nonverbal kommuniziert oder in Einwortsätzen geantwortet. Daher zeigen die MAIN Geschichten von Gagarina und Kolleg/innen (2012, 2019) ein großes Potenzial. Durch den Vergleich der Nacherzählung von Frau Schneider und einem 
früher entstandenen Dialog, in dem sie kaum gesprochen hat, lässt sich vermuten, dass sie die sprachlichen Strukturen trotz der Demenz und ihrer Migration nicht abgebaut hat. Wenn sie durch Aufgaben, wie die Nacherzählung der MAIN Geschichte gefordert wird, ist sie noch in der Lage, in längeren Sätzen zu sprechen und zusammenhängende Gedanken zu äußern.

Das Feld der Mehrsprachigkeit im Zusammenhang mit Demenz ist bis heute sehr wenig erforscht. Daher können die Ergebnisse meiner Arbeit einen Anreiz bieten, in diesem Bereich weitere Forschung zu betreiben. Denn es zeigt, dass demenzerkrankte Menschen zwar wenig sprechen, was jedoch nicht bedeutet, dass sie die Fähigkeit nicht beherrschen. Es fehlt ihnen vielleicht nur der passende Anreiz.

\section{Literatur}

Albert, R., \& Marx, N. (2016). Empirisches Arbeiten in Linguistik und Sprachlehrforschung: Anleitung zu quantitativen Studien von der Planungsphase bis zum Forschungsbericht. Narr Francke Attempto Verlag.

Ash, S., Evans, E., O’Shea, J., Powers, J., Boller, A., Weinberg, D., Haley, J., McMillan, C., Irwin, D., Rascovsky, K., \& Grossman, M. (2013). Differentiating primary progressive aphasias in a brief sample of connected speech. Neurology, 81(4), 329-336. https://doi. org/10.1212/WNL.0b013e31829c5d0e.

Clasmeier, C. (2015). Die mentale Repräsentation von Aspektpartnerschaften russischer Verben. [Dissertation zum Erwerb des Doktorgrades Ruhr-Universität Bochum]. Leipzig.

Demenz und Migration. (2018). http://www.demenz-und-migration.de/demenz-und-migrat ion/. Zugegriffen: 26. Mar. 2018.

Friesen, N. (2020). Die MAIN-Geschichte als Erhebungsinstrument linguistischer Fähigkeiten bei Menschen mit Demenz - ein Überblick. [Unveröffentlichte Masterarbeit]. Ruhr-Universität Bochum.

Gagarina, N., Klassert, A., \& Topaj, N. (2010). Sprachstandstest Russisch für mehrsprachige Kinder. ZAS Papers in Linguistics, 54, 4-55.

Gagarina, N., Klop, D., Kunnari, S., Tantele, K., Välimaa, T., Balčiūnienė, I., Bohnacker, U., \& Walters, J. (2012). MAIN: Multilingual Assessment Instrument for Narratives. ZAS Papers in Linguistics, 56, 1-140.

Gagarina, N., Klop, D., Kunnari, S., Tantele, K., Välimaa, T., Bohnacker, U., \& Walters, J. (2019). MAIN: Multilingual Assessment Instrument for Narratives - Revised. ZAS Papers in Linguistics, 63.

Goodglass, H., Kaplan, E., \& Weintraub, S. (1983). Boston Naming Test. Lea \& Febiger.

Hamers, J. F., \& Blanc, M. (2000). Bilinguality and bilingualism. Bilingualité et bilinguisme (2. Aufl.). Cambridge University Press.

Hier, D. B., Hagenlocker, K., \& Shindler, A. G. (1985). Language disintegration in Dementia: Effects of etiology and severity. Brain and Language, 25(1), 117-133. https://doi.org/10. 1016/0093-934x(85)90124-5. 
Luckabauer, F. (2011). Demenzielle Auswirkungen auf Sprache und Gedächtnisstrukturen - Frontotemporale Demenz und Alzheimer im Vergleich. AV Akademiker Verlag.

Meitner, K. (2019). Sprachproduktion und Raumwahrnehmung bei Alzheimer-Demenz: Ein systematischer Vergleich zum gesunden kognitiven Altern mittels Eye-Tracking. [Unveröffentlichte Masterarbeit] Technische Universität Dortmund. http://www.divers itaet.fb15.tu-dortmund.de/cms/de/Projekte-und-Labore/psycholinguistics-laboratories/ PL-Medienpool/MA_Meitner-Kim.pdf.

Neppert, D. (2010). MigrantInnen und Demenz-Ressourcen, Belastungen und Krankheitskonzepte: Eine Rekonstruktion anhand von Interviews mit VertreterInnen von MigrantInnenselbstorganisationen [Master Arbeit, Fachhochschule Kiel]. https://www.fh-kiel.de/fil eadmin/data/sug/pdf-dokument/neppert/gesamtthesisfinale.pdf.

Nicholas, M., Obler, K. L., Albert, M. L., \& Helm-Estabrooks, N. (1985). Empty speech in Alzheimers's disease and fluent aphasia. Journal of Speech and Hearing Research, 28(3), 405-410. https://doi.org/10.1044/jshr.2803.405.

Sachweh, S. (2000). „Schätzle hinsitze!“ Kommunikation in der Altenpflege. Lang.

Sachweh, S. (2003). ,,so frau adams $\downarrow$ guck mal $\downarrow$ ein feines bac-spray $\downarrow$ gut. Charakteristische Merkmale der Kommunikation zwischen Pflegepersonal und Bewohnerinnen in der Altenpflege. In R. Fiehler, \& C. Thimm (Hrsg.), Sprache und Kommunikation im Alter (S. 143-160). Verlag für Gesprächsforschung.

Open Access Dieses Kapitel wird unter der Creative Commons Namensnennung 4.0 International Lizenz (http://creativecommons.org/licenses/by/4.0/deed.de) veröffentlicht, welche die Nutzung, Vervielfältigung, Bearbeitung, Verbreitung und Wiedergabe in jeglichem Medium und Format erlaubt, sofern Sie den/die ursprünglichen Autor(en) und die Quelle ordnungsgemäß nennen, einen Link zur Creative Commons Lizenz beifügen und angeben, ob Änderungen vorgenommen wurden.

Die in diesem Kapitel enthaltenen Bilder und sonstiges Drittmaterial unterliegen ebenfalls der genannten Creative Commons Lizenz, sofern sich aus der Abbildungslegende nichts anderes ergibt. Sofern das betreffende Material nicht unter der genannten Creative Commons Lizenz steht und die betreffende Handlung nicht nach gesetzlichen Vorschriften erlaubt ist, ist für die oben aufgeführten Weiterverwendungen des Materials die Einwilligung des jeweiligen Rechteinhabers einzuholen.

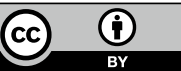

